\title{
Stadt ohne Gott oder Die Rückkehr des Heiligen?*
}

\author{
Albrecht Grözinger
}

\section{Stadt ohne Gott - vom Schicksal einer Illusion}

Ein möglicher Einstieg in das Thema wie die Grundlinien meiner Überlegungen wären vor 30 Jahren ganz klar vorgezeichnet gewesen. Ich hätte mit groBer Wahrscheinlichkeit begonnen mit einem Hinweis auf das Buch von Harvey Cox "Stadt ohne Gott?" und hätte den Thesen dieses Buches zugestimmt. Ich beginne mit einem Hinweis auf dieses Buch, werde mich jedoch sehr viel differenzierter zu den Thesen dieses Buches äußern, als ich dies vor dreißig Jahren getan hätte.

Es macht immer noch guten Sinn, auch im ersten Jahrzehnt des 21. Jahrhunderts an das Buch von Harvey Cox zu erinnern, das im Jahre 1965 zum ersten Mal in englischer und kurz danach in deutscher Sprache erschienen ist. Harvey Cox ist Theologieprofessor an der Harvard Divinity School und gehört zu denjenigen amerikanischen Theologen, die dem Zusammenhang von Religion, Kultur und Gesellschaft besonders intensiv nachgespürt haben. Der amerikanische Originaltitel des Buches lautet ins Deutsche übertragen "Die säkulare Stadt. Säkularisation und Urbanisation in theologischer Perspektive“. In Deutschland ist das Buch unter dem etwas reißerischen aber prägnanten Titel "Stadt ohne Gott?" erschienen. Das Buch hat mehrere Generationen von Theologinnen und Theologen vor allem in der westlichen Hemisphäre in ihrem Nachdenken über den Ort der Religion in den modernen pluralistischen Gesellschaften geprägt. Meine Generation hat dieses Buch bereits im ersten Semester des Theologiestudiums begeistert gelesen. Es hat uns damals inspiriert und schien uns den Weg in die Zukunft zu öffnen.

Harvey Cox zeichnet in seinem Buch ein eindrückliches Bild der Stadt in der Moderne. Wie ein Fanfarenstoß muten die ersten Sätze seines Buches an: „Die Heraufkunft einer urbanen Zivilisation und der Zusammenbruch der traditionellen Religionen sind die beiden bestimmenden Kennzeichen unserer Zeit ... Heute steht die säkularisierte Großstadt sowohl als Muster unseres Zusammenlebens da wie als Symbol unseres Weltverständnisses. Wenn die Griechen den Kosmos als eine ins Unendliche ausgeweitete Polis begriffen, wenn der mittelalterliche Mensch ihn als ein unendlich vergrößertes Feudalschloss sah, so begreifen wir heute das Universum als die Stadt des Menschen. Es ist ein Feld menschlicher Entdeckung und Bemühung, aus dem die Götter geflohen sind. Die Welt ist zur Aufgabe des Menschen geworden und seiner Verantwortung übergeben. Der moderne Mensch ist Kosmopolit. Die Welt ist seine Stadt geworden, und seine Stadt hat sich zur Welt erweitert. Der Prozess, der dies in Szene gesetzt hat, wird von uns Säkularisierung genannt ... Das Zeitalter der säkularisierten Stadt, eine Epoche, deren innere Einstellung sich rapid bis

- Vortrag vor dem Pfarrkonvent der Vereinigten Kirchenkreise Dortmund am 24. Oktober 2001. 


\section{Thema: Säkularisierung}

in die letzte Ecke der Welt ausbreitet, ist ein Zeitalter der völligen ,Religionslosigkeit'. Es lassen sich die Fragen der Moral oder des Lebenssinns nicht länger durch religiöse Regeln oder Ritualien beantworten ... Die Säkularisierung ist in Gang, und wenn wir überhaupt unsere Zeit verstehen und auf sie eingehen wollen, müssen wir lernen, sie in ihrer unaufhaltsamen Säkularisierung zu lieben. ${ }^{\mu 1}$

So weit Harvey Cox im Jahre 1965. Ich denke, man kann auch noch heute etwas von der Faszination begreifen, die damals für uns junge Theologen und Theologinnen von diesen Sätzen ausgingen. Und man wird diesen Sätzen und dem Buch von Harvey Cox sicher nicht dadurch gerecht, dass wir sie lediglich als das Produkt einer säkularistisch überhitzten Epoche im Zusammenhang der sich anbahnenden Studentenrevolte der ausgehenden $60 \mathrm{er}-J$ ahre des vergangenen Jahrhunderts verstehen. Dazu steht in dem Buch zu viel an Richtigem und auch Unabgegoltenem. Zugleich ist jedoch unübersehbar, dass in das Buch grandiose Irrtümer eingeschrieben sind, was die Zukunft der Religion anbetrifft.

Cox hat bereits im Jahre 1965 hellsichtig erkannt, dass der Vorgang der Verstädterung unserer Welt-Gesellschaft ein umfassender kultureller Vorgang ist, der in seiner Tragweite kaum überschätzt werden kann. Wenn wir heute von

\section{Globalisierung und Urbanisierung produzieren Reiigion.} unserer weit als einem, global village' sprechen, so bekommt Harvey Cox in einem Ausmaße Recht mit dem er bei Entstehung seines Buches wohl selbst nicht gerechnet hat. Die mediale und ökonomische Vernetzung der Welt hat sich nach dem Zusammenbruch der unter dem Einfluss der Sowjetunion stehenden ,sozialistischen' Hemisphäre beschleunigt und intensiviert. Immer mehr Menschen wohnen in und an den Rändern der großen urbanen Siedlungsräume unseres Planeten, die Tag für Tag immer noch anwachsen. Diese Veränderungen als eine kulturelle Herausforderung begriffen zu haben, ist das bleibende Verdienst des Buches von Harvey Cox. Geirrt hat sich Cox darin, dass er kulturelle Urbanisierung und Globalisierung nahezu bruchlos mit einem Abnehmen der Religiosität in diesem neu entstehenden globalen Kulturraum gleichgesetzt hat. Heute können wir beobachten, dass diese gegenläufige Korrelation mit Sicherheit eine Fehlprognose war. Religion boomt heute geradezu. Urbanisierung und Globalisierung sind in ihren Folgen weniger religionskritisch oder religionszersetzend als vielmehr religionsproduktiv. Globalisierung und Urbanisierung produzieren Religion.

Nichts wäre nun falscher, als wenn Theologinnen und Theologen sich angesichts dieser Perspektive einfach selbstzufrieden und selbstgewiss zurücklehnten. Denn der gegenwärtige Boom der Religion ist ja nichts Eindeutiges. Er ist in sich sehr differenziert, widersprüchlich und ambivalent. Wir können in den USA sehen, dass eine multikulturelle Gesellschaft nicht das Ende der überlieferten Religionsformen bedeutet. Die USA sind in einem Maße kirch-

1 Harvey Cox: Stadt ohne Gott?, Stuttgart/Berlin ${ }^{5} 1969,10 \mathrm{f}$. 
lich, wie wir uns das in Europa kaum noch vorstellen können. Wir erleben eine Renaissance des Islam im Nahen und Mittleren Osten. Wir sehen in Europa neue Formen von religiösen Empfindungen und Lebensäußerungen wachsen. Allerdings ist dieser ,Boom 'von Religion nur dann richtig ins Auge gefasst, wenn wir auch die jeweiligen Umstände dieses Religions-Booms nicht außer Acht lassen. Ob wir etwa die ,Electronic-Churches' der USA, die Millionen von Menschen faszinieren und anziehen, auf dem europäischen Kontinent wirklich wollen, darüber wäre sehr eingehend zu sprechen. Der Boom des Islam ist auch verbunden mit einem religiösen Fundamentalismus, der gegenwärtig die weltpolitischen Konstellationen neu formiert. Dass in den kriegerischen Auseinandersetzungen unserer Gegenwart, wie auf dem Balkan oder im Konflikt zwischen Israel und den Palästinensern, die Religion wieder zunehmend eine Rolle spielt, ist für mich alles andere als Anlass zur Genugtuung. Und die freien Religionsformen in den pluralistischen und zunehmend multikulturellen Gesellschaften Europas haben einen Markt der Religionen und Weltanschauungen entstehen lassen, auf dem es immer schwerer wird, zwischen Ernsthaftigkeit und Scharlatanerie, zwischen Lebensdienlichkeit und Lebendfeindlichkeit bestimmter religiöser Formen und Rituale zu unterscheiden. Ein Religions-Boom an sich sagt noch nichts darüber aus, wie ein solches Phänomen in seinen Wirkungen dann wirklich zu bewerten ist.

\section{Welche Religion passt zum Protestantismus?}

Genau an dieser Stelle sind Theologie und Kirchen herausgefordert. Ich möchte diese Herausforderung, wie ich sie sehe, in wenigen Strichen skizzieren. Es erscheint mir wenig sinnvoll, weiterhin über Religion an sich zu reden. An der Frage, ob eine Gesellschaft religiös grundiert ist oder nicht, lässt sich $\mathrm{m}$. E. recht wenig ablesen. Wir haben hochreligiöse Gesellschaften, wie etwa in Nordirland, die mit einem gewaltigen Konfliktpotenzial und mit der damit verbundenen Bereitschaft, Gewalt auch auszuüben, zu leben haben. Und wir haben weitgehend säkularisierte Gesellschaften, wie etwa in den Niederlanden, die sich durch ein hohes Toleranzprofil und eine weit verbreitete Sensibilität für Fragen der Menschenrechte und des Minderheitenschutzes auszeichnen. Wir werden also auf keinen Fall sagen können, dass religiös grundierte Gesellschaften auf jeden Fall ,bessere', humanere Gesellschaften sind als Gesellschaften mit einem, geschrumpften' Bestand an religiösen Traditionen und an praktizierter Religion. Dies hängt nicht zuletzt damit zusammen, dass ,Religion' ein durch und durch ambivalentes Phänomen ist. Über Religion lassen sich Aggressionen ebenso mobilisieren wie karitative Empathie. Religion kann Freiheitsräume einengen wie Freiheitsräume schaffen. Deshalb stehen Theologie und Kirche vor der Aufgabe, ein qualitatives Verständnis von Religion zu entwickeln und sich von einem solchen qualitativen Verständnis von Religion in ihrer Praxis leiten zu lassen. Praktisch-theologisch relevant ist in erster Linie nicht die Frage: ob Religion oder nicht, sondern die Frage: welche Religion. Und deshalb frage ich nun in einem 


\section{Thema: Säkularisierung}

nächsten Schritt: Von welcher Religion sollen sich protestantische Theologie und protestantische Kirchen bestimmen lassen? Oder noch zugespitzter: Welche Religion passt zum Protestantismus?

Diese Frage werden wir heute nicht mehr allein dogmatisch-deduktiv beantworten können. Religion lassen sich die Menschen heute nicht mehr zu-sprechen. Religion wird heute von den Menschen autonom gelebt. Deshalb tun

Religion wird heute von den Menschen autonom gelebt. wir gut daran, den Formen von Religion, wie wir sie heute alltagsweltlich antreffen, sensibel wahrzunehmen und nachzuspüren. Ein praktischtheologisch verantwortlicher wie aussagekräftiger Religions-Begriff wird nur noch durch ein Ineinander von induktiver Wahrnehmung und systematischer Reflexion dieser Wahrnehmung bestehen können. Und genau an dieser Stelle wird für mich das Buch von Harvey Cox wiederum relevant. Harvey Cox hatte ja vor nun beinahe vierzig Jahren den Versuch unternommen, anhand der Wahrnehmung gelebter Urbanität auch die gelebte Religion der Menschen beziehungsweise deren Verlust ins Auge zu bekommen. Das Defizit des Buches bestand nicht in diesem theoretischen Setting, sondern in der überzogenen Prognose, beziehungsweise in dem bruchlosen Fortschreiben einer Entwicklung, die Cox zu einem bestimmten Zeitpunkt beschrieben hat. Deshalb möchte ich aus den Stärken und Schwächen dieses Buches lernen - quasi auch als eine kleine Hommage an dieses Buch, das mich während meines Theologiestudiums so fasziniert hat. Ich werde also weniger prognostisch, sondern verstärkt phänomenologisch vorgehen. Ich versuche eine Augenblicks-Beschrejbung gelebter Religion in den urbanen Gebieten unserer europäischen Gegenwart. Aber ich beschreibe nicht nur. Ich werde versuchen angesichts dieser von mir beschriebenen urbanen Religion diese urbane Religion als Herausforderung für die theologische Reflexion und kirchliche Praxis zu begreifen, also genau das von mir postulierte Ineinander von induktiver Beschreibung und theologischer Reflexion zu erfüllen. ${ }^{2}$

Harvey Cox hat in seinem Buch zu Recht darauf verwiesen, dass in jede urbane Kultur bestimmte religiöse Erfahrungen und Religionsformen eingeschrieben sind, auch wenn sie oft gar nicht mehr als explizit religiös erscheinen. Die griechische Polis war schon im Stadtbild durch die strenge Trennung der ökonomisch-politischen und der religiösen Sphäre gekennzeichnet. Der Tempel und der Markt mit der Agora waren architektonisch strikt voneinander geschieden. Nur in einem Akt der übergänglichen Begehung waren die beiden Bereiche für die Menschen zugänglich. Die mittelalterliche Stadt war demgegenüber geprägt durch die Zentralstellung der Kathedrale mit ihrer architektonisch klaren Ästhetik, die sich von dem umgebenden Gewimmel der kleinen Straßen und Gassen klar abhob. Jeder und jede, die in diesen Städten wohnten, wusste wo die wahre Mitte des städtischen Lebens zu sein hatte. Die Stadt der Renaissance entthronte die Kathedrale und setzte den Markt und das Rathaus in die Mitte der Stadt. Architektonische Säkularisierung

2 Vgl, dazu ausführlicher Albrecht Grözinger: Kirche im Zeitalter der Globalisierung, Waltrop 2002. 
könnte man diesen Vorgang nennen. Mit jedem dieser städteplanerischen Paradigmenwechseln waren auch jedes Mal Verschiebungen im Lebensgefühl der Menschen, die diese Städte bewohnten, verbunden, Stadtarchitektur ist immer auch Ausdruck des impliziten und expliziten Lebensentwurfs der Menschen.

In welches Lebensgefühl weist uns die Stadtarchitektur unserer Gegenwart ein? Zunächst einmal dürfte der Blick zurück und ein Vergleich mit den 70erund frühen $80 \mathrm{er}-J a h r e n$ des vergangenen Jahrhunderts aufschlussreich sein. Jürgen Habermas hat bei Gelegenheit zu Recht darauf hingewiesen, dass die entscheidenden Veränderungen im Bild der deutschen Städte nicht durch die verheerenden Katastrophen der Bombennächte des 2. Weltkriegs verursacht wurden, sondern dass dies erst nach dem Zweiten Weltkrieg geschah. Nach dem Zweiten Weltkrieg wurde in Deutschland mehr alte Bausubstanz beseitigt als durch die Kriegszerstörungen selbst. Das Ideal dieses städtebaulichen Kahlschlags war die durchrationalisierte, die ökonomische Stadt. Die Stadtautobahnen, die auch heute noch das Bild unserer Städte bestimmen, waren der exemplarische Ausdruck dieses rigorosen Planungswillens. Auf der einen Seite soliten die Bereiche von Produktion, Konsum und Wohnen klar voneinander getrennt sein. Auf der anderen Seite mussten sie wieder schnell gegenseitig erreichbar sein. Das städtebauliche Regulativ war die jeweilige Effizienz eines urbanen Raumes, die Optimierung der Funktionen des Produzierens, des Konsumierens und der körperlichen Regeneration. Insofern war dieses städtebauliche Konzept der Ausdruck einer Gesellschaft, die die kargen Zeiten des Krieges so schnell wie möglich hinter sich lassen wollte und den neu erworbenen materiellen Reichtum optimal produzieren und optimal konsumieren wollte. Es war, wie wenn Ludwig Erhard und Mies van der Rohe in eine etwas unheilige Allianz getreten wären.

Spätestens gegen Ende der 80er-Jahre war absehbar, dass dieses städtebauliche Konzept an sein Ende gelangt war. Und zwar deshalb, weil es ganz offensichtlich die Bedürfnisse der Menschen nicht mehr zu befriedigen vermochte. Wie sah das Neue aus, das an die Stelle des Alten trat? Ich nenne zunächst einmal einige Stichworte und versuche dann so etwas wie eine Gesamtschau der neueren städtebaulichen Tendenzen.

Stadtarchitektur ist immer auch Ausdruck des Lebensentwurfs der Menschen.

Die Stichworte lauten: Museums-Boom, verkehrsberuhigte Zonen, Neue Plätze und Passagen, ökologisches Bauen. Worin sind diese neuen Akzente begründet? Es ist sicher nicht so, dass die Frage nach der ökonomischen Effizienz verschwunden wäre. Es wäre blauäugig zu behaupten, die Ökonomie bestimmte nicht mehr die städtebaulichen Grundentscheidungen. Das Gegenteil ist der Fall. Neu ist allerdings, dass der Gesichtspunkt der Ökonomie nicht mehr der alleinige Faktor bei städtebaulichen Grundentscheidungen st. Nichts geht ohne Ökonomie, aber die Ökonomie ihrerseits muss sich nun Jurch andere ,Werte' legitimieren. Man könnte etwas abgekürzt, aber damit sicher nicht falsch sagen: Im Städtebau der letzten zwanzig Jahren wurde ei' alte Weisheit neu entdeckt. Diese alte Weisheit, die zudem eine biblische 


\section{Thema: Säkularisierung}

Weisheit ist, lautet: Der Mensch lebt nicht vom Brot allein. Die Menschen entdeckten sich neu in ihren sozialen, geselligen und kulturellen Bedürfnissen. Deshalb werden plötzlich die Museen wieder wichtig. Deshalb braucht es menschenfreundliche Plätze der Begegnung; Plätze, auf denen man weilen kann, und die man nicht lediglich überquert. Die Menschen wollen ihre Städte wieder beheimatend erfahren. Und sie merken zugleich, dass sie sich in ihren Städten selbst begegnen, dass der jeweilige Städtebau Auskunft gibt über die Conditio humana, wie sie den Menschen in ihrer Gegenwart aufscheint.

Exemplarisch wird dieser Vorgang konkret an der städtebaulichen Rekonstruktion und Neukonstruktion Berlins nach der Vereinigung der beiden deutschen Nachkriegsstaaten. Der Potsdamer Platz und die Friedrichsstraße spielen dabei eine entscheidende Rolle. Die Friedrichsstraße - so das mit

Das Passagen-Bedürfnis ist großem Pathos vorgetragene architektonische offensichtlich verbreitet. Programm - soll wieder zur Flanier- und Passagenstraße werden. So weit so gut. Nur war die Friedrichstraße im Gegensatz etwa zum Kurfürstendamm nie eine Flanierstraße, und Passagen kannte das Vorkriegsberlin im Vergleich zu Paris oder London kaum. Was im breiten Bewusstsein als historische Wiederherstellung gilt, ist im Grunde nichts anderes als eine Neukonstruktion auf Grund eines kollektiven Bedürfnisses. Das Passagen-Bedürfnis ist offensichtlich so verbreitet, dass es sich die Passagen immer dort schafft, wo die Chance dazu besteht.

Welche Erfahrung meldet sich nun an in dem, was ich hier das Passage-Bedürfnis genannt habe? Es ist die Erfahrung der Mehrdimensionalität der Conditio humana: Die Erfahrung nämlich, dass wir Menschen an unserer Menschlichkeit Schaden nehmen, wenn wir in unserem Menschsein auf eine Funktion festgelegt oder gar reduziert werden. Wir Menschen sind mehr als Konsumenten. Wir Menschen sind mehr als freie Kräfte auf dem Spiel des Arbeitsmarktes. Wir Menschen sind aber auch immer mehr als Partner und Partnerinnen in Beziehungen, als Theaterbesucherinnen und -besucher, auch mehr als Mitglieder einer Kirche. Der Mensch begegnet sich in der Passage als der Mensch in seinem Nicht-fest-gelegt-Sein, in seiner Übergängigkeit. Der Mensch sucht und begegnet sich in seinem Geheimnis.

Und dies ist genau der Ort, wo die Religion ins Spiel kommt. Wenn die Städte unserer Gegenwart nicht mehr allein Stätten der ökonomischen Effizienz sind, sondern Stätten menschlicher Selbst-Erkundung und menschlicher SelbstDeutung, dann werden die Museen mit ihren Bildern und Symbolen zu Kultstätten des postmodernen Menschen. Dann werden die Passagen und Orte zu rituellen Stätten der Selbst-Begegnung und Selbst-Findung. Und deshalb können wir mit gutem Grund davon sprechen, dass in unsere Städte das ,Heilige' als Grundsymbol des menschlichen Geheimnisses zurückgekehrt ist. Und genau dies hat Harvey Cox vor vierzig Jahren nicht vorausgesehen, nämlich diese Rückkehr des Heiligen in die Stadt, wie sie in der Architektur und auch in der Literatur unserer Gegenwart unübersehbar ist. 
Der amerikanische Schriftsteller Paul Auster ist der exemplarische Autor dieser neuen Stadt-Erfahrung. Seine Romane beschreiben und verrätseln zugleich die postmoderne Stadt-Erfahrung. Das Labyrinth der urbanen Metropole wird in seinen Romanen transparent hin zur Geheimnisstruktur des Lebens schlechthin. Aufschlussreich und anschaulich sind in diesem Zusammenhang die Schlusssätze des Romans "Moon Palace“: "Mein fünftes Paar Schuhe kaufte ich mir am 3. Januar 1972 in einem Ort namens Lake Elsinore. Drei Tage später stieg ich, fix und fertig vor Erschöpfung, mit 413 Dollar in der Tasche über die Hügel nach Laguna Beach hinunter. Vom Gipfel des Vorgebirges aus konnte ich bereits den Ozean sehen, aber ich ging weiter, bis ich ganz unten am Wasser war. Es war vier Uhr nachmittags, als ich meine Schuhe auszog und den Sand an meinen Fußsohlen spürte. Ich hatte das Ende der Welt erreicht, dahinter waren nur noch Luft und Wellen, eine Leere, die sich bis an die Küsten von China erstreckte. Hier fange ich an, sagte ich zu mir, hier soll mein Leben anfangen.

Ich stand lange am Strand, wartete, bis die letzten Strahlen der Sonne verschwunden waren. Hinter mir ging die Stadt ihren Geschäften nach und machte die vertrauten amerikanischen Geräusche des ausgehenden Jahrhunderts. Ich blickte dem Bogen der Küste entlang und sah eins nach dem anderen die Lichter in den Häusern angehen. Dann stieg der Mond hinter den Hügeln empor. Es war Vollmond, gelb und rund wie ein glühender Stein. Ich folgte ihm mit den Augen auf seinem Weg in den nächtlichen Himmel und wandte mich erst $a b$, als er seinen Platz in der Dunkelheit gefunden hatte. ${ }^{\prime 3}$ Dies ist die postmoderne Erfahrung des ,Heiligen', die Erfahrung des fascinosum tremendum, wie es Rudolf Otto in seiner berühmten Studie über "Das Heilige" phänomenologisch beschrieben hat. Aus der Stadt ohne Gott ist die Stadt geworden, in die ganz offensichtlich das Heilige oder zumindest eine Ahnung davon wieder zurückgekehrt ist.

\section{Protestantische Religion}

Es wäre nun eine Illusion, aus dieser Feststellung über die Rückkehr des Heiligen in die postmoderne Stadt den Schluss zu ziehen, Kirche und Theologie konnten ihre Inhalte unverwandelt und in den traditionellen Beteiligungsformen an die Menschen weitergeben.

Religion unter den Bedingungen der Postmoderne unterscheidet sich wesentlich auch noch von der Religion der klassischen Moderne. Die Menschen finden sich heute auf einem vielfältigen und heterogenen Markt der Religionen und Weltanschauungen vor. Auf diesem Markt sehen sich die Menschen um und greifen nach dem, was ihnen selbst für ihr Leben lebensdienlich erscheint. Dabei erstellen sie sich die Kriterien dieser Lebensdienlichkeit jeweils individuell für sich selbst. Sie lassen sich solche Kriterien nicht mehr vorschreiben, wenn sich auch diese Kriterien nicht ohne äußere Beeinflus-

Poul Auster: Mond über Manhattan, Reinbek 1992, 392. 


\section{Thema: Säkularisierung}

sung herausbilden. So formt sich heute Lebensgeschichte in einem beinahe schon eingespielten lebensweltlichen Synkretismus heraus, der auch das religiöse Selbstverständnis und die religiöse Praxis der Menschen nicht unbeein. flusst lässt.

Darauf haben sich Theologie und Kirche einzustellen - ob sie dies nun wollen oder nicht. Und zwar illusionslos einzustellen. So erzeugt es unter Pfarrerin. nen und Pfarrern immer wieder eine Mischung aus Verwunderung und Kopfschütteln, wenn empirische Umfragen belegen, dass ein nicht geringer Teil der regelmäßigen Gottesdienstbesucherinnen und -besucher fernöstliche Reinkarnationsvorstellungen in ihr individuelles Weltbild zu integrieren vermögen. ${ }^{4}$ Ein Synkretismus, der aus der theologischen Warte heraus betrachtet nicht korrekt sein mag, den aber die Menschen ganz selbstverständlich in Anspruch nehmen. Die Menschen lassen sich von Kirche und Theologie nicht mehr sagen, wie sie ihr Leben zu führen haben und welche religiöse Bilder und Symbole sie für ihre Lebensgeschichte in Anspruch nehmen. Wohl aber

\section{In der Postmoderne wird die} synkretistische ,Ketzerei` zum Normalfall der Religion. erwarten die Menschen von Kirche und Theologie - auch das belegen empirische Umfragen -, dass Kirche und Theologie den Menschen solche Bilder und Symbole zur Verfügung stellt, die sie dann in eigener Entscheidung in ihr Leben integrieren oder nicht. Wahrscheinlich ist dieser Vorgang so neu nicht. Menschen mögen schon immer mehr oder weniger auf diese Weise mit der kirchlichen Tradition verfahren sein. Allerdings geschah dies eher untergründig, quasi ,ketzerisch', stets mit dem Verdacht des theologisch Inkorrekten konfrontiert. In der Postmoderne wird nun die lebensgeschichtlich-synkretistische ,Ketzerei' zum Normalfall der Religion. Darauf haben sich Kirche und Theologie nüchtern einzustellen. Und sie brauchen m. E. diese ,Ketzerei' auch nicht zu fürchten. Denn der lebensgeschichtliche Synkretismus ist die Basis, die die Nachfrage nach religiösen Bildern und Symbolen erst erzeugt. Kein Mensch kann den eigenen Bedarf an Bildern, Symbolen und Geschichten, den er benötigt, um seine eigene Lebensgeschichte zu verstehen und zu gestalten, aus sich selbst heraus freisetzen. Die Menschen sind auf solche, fremden' Ressourcen an Bildern, Symbolen und Geschichten angewiesen. Diese Ressource ist heute jedoch nicht mehr monopolisiert durch eine einzige Religion oder Weltanschauung, sondern diese Ressourcen sind auf einem bunten und vielfältigen Markt zu finden.

Für Kirche und Theologie kommt es darauf an, auf diesem Markt ihre Kenntlichkeit zu gewinnen. Darin besteht für mich die Herausforderung der Postmoderne. Nicht darin, die postmoderne Vielfalt zu unterlaufen oder gar zu reduzieren, was ohnehin ein müßiges Geschäft wäre. Sondern in der postmodernen Vielfalt das eigene Profil zu schärfen und für andere kenntlich zu machen. Wie dies geschieht, möchte ich gerne andeuten, indem ich vier

4 Vgl. dazu exemplarisch Manfred Bruhn / Albrecht Grözinger (Hg.): Kirche und Marktorientle rung. Impulse aus der Ökumenischen Basler Kirchenstudie, Freiburg/Schweiz 2000, bes. 169-184. 
Punkte entfalte, an denen protestantische Religion ihre Kenntlichkeit gewinnen und entfalten kann.

\section{1. Übergänglichkeit}

Ich habe in meiner Analyse unserer kulturellen Gegenwart das Passagen-Bedürfnis der Menschen als ein zentrales Bedürfnis bezeichnet. Darin meldet sich der Wunsch der Menschen an, ihrer eigenen Lebensgeschichte ansichtig zu werden. Und zwar in der Weise ansichtig zu werden, dass ihr Leben als ein Leben erscheint, dass nicht in bloßen Funktionen aufgeht. ,Übergänglichkeit' meint: Ich bin immer mehr, als ich tue. Ich möchte auch anders sein, als das, was mir an Rollen zugeschrieben wird. Insofern meldet sich hier ein elementares humanes Bedürfnis zu Wort, das in seinem kritischen Gehalt gegen alle ökonomischen und weltanschaulichen Ansprüche, mit denen wir ständig konfrontiert werden, nicht genug gewürdigt werden kann. Deshalb bedarf dieses Bedürfnis der Hege und Pflege, und dies heißt nicht zuletzt: der verantwortlichen und reflektierten Gestaltung. Protestantische Religion - so meine These - steht für diese Wahrnehmung und Gestaltung der. Übergänglichkeit des Lebens. Bei jeder Taufe, bei jeder Hochzeit, auch am Grabe wird die Übergänglichkeit menschlichen Lebens thematisch. Und sie wird dies in einem spezifischen Licht: nämlich der Zusage der vorbehaltlosen Annahme des menschlichen Lebens durch Gott. Diese Zusage wird von den Menschen dann gehört, wenn sie ihnen nicht als dogmatisches Korsett angepasst wird, sondern wenn sie diese Zusage als ein Element hören können, das sie in ihr individuelles unverwechselbares Leben zu integrieren vermögen. Im Grunde wird damit jede Predigt, auch die sonntägliche Predigt, zur Kasual-Predigt. Die Kasualie ist der Normalfall der Kirche in der Postmoderne.

\section{Rettung der Utopie}

Das 20. Jahrhundert hat die Menschen in Europa gründlich desillusioniert. Europa hatte die Schwelle zu diesem 20. Jahrhundert mit einem ungestümen Optimismus überschritten, der weit auch in Kirche und Theologie hinein ausstrahlte. Dieser Optimismus wurde auf den Schlachtfeldern des Ersten und Zweiten Weltkriegs, durch Auschwitz und den Archipel Gulag zunichte. Das Vertrauen in den technologischen Fortschritt wurde durch Hiroshima und Tschernobyl fraglich. Und wir sind gegenwärtig dabei zu sehen, wie die letzte Heilsverheißung des 20. Jahrhunderts, nämlich das Vertrauen in die globalisierte Ökonomie und in ständig boomende Aktienmärkte ihre ersten Risse bekommt. Dieses Vertrauen war in den meisten Fällen getragen von einer geschlossenen Weltanschauung, die die entsprechenden Entscheidungen und Handlungen legitimierte. Der Nationalismus schickte die Menschen auf die Schlachtfelder des ersten Weltkriegs. Der Kommunismus machte den Archipel Gulag möglich. Die Rassentheorie des Nationalsozialismus ebnete dem Weg zum Völkermord. Der wissenschaftlich-technischen Entwicklung wurde ein in- 


\section{Thema: Säkularisierung}

härenter Fortschritt unterstellt, der ihre Risiken noch allemal auszugleichen vermag. Nicht zu Unrecht hat man die Utopie als den geheimen Motor dieser geschlossenen Ideologien identifiziert. Der Kommunismus lebte von der Utopie der klassenlosen Gesellschaft, der Nationalsozialismus von der Utopie der Volksgemeinschaft, der neuzeitliche Fortschrittsgedanken in Wissenschaft und Ökonomie von der Utopie des größtmöglichen Glückes aller. Und mit dem Zerbrechen der geschlossenen Ideologien des 20. Jahrhundert ist auch die Utopie in Verdacht geraten. Der französische Philosoph Jean-François Lyotard hat in diesem Zusammenhang vom ,Ende der großen Erzählungen' gesprochen. Wer mit jungen Menschen spricht, merkt dies sofort: Nichts erzeugt bei ihnen größere Vorbehalte als utopisches Denken. Und dieser Vorbehalt hat ja auch seine geschichtliche Berechtigung. Wo Utopien realisiert werden, wird die Wirklichkeit, die der Utopie entgegensteht, zweitrangig. Im besten Fall wird eine widerständige Wirklichkeit ignoriert. Im schlimmsten Fall wird sie gewaltsam beseitigt. ,Wo gehobelt wird, da fallen Späne' - in dieser Sentenz finden so unterschiedliche Gestalten wie Adam Smith, Karl Marx und Sloboda Mi. losevic sehr schnell ihre Gemeinsamkeit. Nein, der Verdacht gegen die Utopie speist sich nicht zuletzt aus einer geschichtlichen Desillusionierung, der ein durchaus humaner Gehalt innewohnt.

Allerdings geraten wir mit dem Verdacht gegen die Utopie in ein gewisses Di. lemma. Die Utopie ist zu Recht in den Verdacht geraten. Gleichwohl brauchen wir Menschen Bilder, Symbole und Geschichten, die über unsere gegenwärtige Wirklichkeit hinausragen. Ohne solche Bilder, Symbole und Geschichten würden wir an unserer eigenen Wirklichkeit verkümmern. Wir wären einer ebenso geschichtslosen wie zukunftslosen Gegenwart ausgeliefert, wir würden an uns selbst verkümmern. Welchen Ausweg gibt es aus diesem Dilemma?

Ich plädiere in diesem Zusammenhang für die Rettung der Utopie. Allerdings für eine ganz spezifische Rettung. In den stürmischen Jahren der Studentenbewegung der ausgehenden 60er-Jahre des vergangenen Jahrhunderts galt das Motto: Utopien sind dazu da, realisiert zu werden. Insofern war - dies se-

Ich plädiere für die Rettung der Utopie durch eine Verweigerung ihrer Realisierung. hen wir heute aus dem Abstand heraus noch deutlicher als damals - die Studentenbewegung eine durch und durch utopisch inspirierte Bewegung. Die Utopie war nur dort verdächtig, wo sie sich gegen ihre Realisierung sperrte. Dann galt sie als Illusion, als Lug und Trug. Ich plädiere demgegenüber für die Rettung der Utopie durch eine Verweigerung ihrer Realisierung. Und dies heißt: Es gilt, die Utopie zurückzuholen an ihren ursprünglichen Ort. Und dieser ursprüngliche Ort ist die Kunst und die Religion. Dort gewinnt die Utopie ihre inspirierende Kraft. Und sie wird totalitär, wenn wir die Utopie in den Bereich des Politischen und des Ethischen zu ziehen versuchen, um sie dort zu ,realisieren'. Religion und Kunst bewahren die Utopie vor ihrer totalitären Realisierung. Theologisch gesprochen hieß dies einmal: eschatologischer Vorbehalt.

Lassen Sie mich diese meine These an einem Beispiel erläutern. Kein Mensch kann nach der Bergpredigt leben. Und der Versuch einer geschichtlichen Red- 
lisierung der Bergpredigt hätte einen absolut totalitären Gottesstaat zu Folge. Und gleichwohl kann die Bergpredigt unsere ethische Reflexion und unser Verhalten inspirieren, gerade weil wir wissen, dass wir in dieser Welt nie so leben werden, wie es die Bergpredigt uns vor Augen stellt. Insofern hatte vielleicht Bismarck mit seinem Satz, mit der Bergpredigt lasse es sich nicht regieren, einen größeren Respekt vor der Bergpredigt als diejenigen, die versuchen, die Bergpredigt in kleine ethische Alltags-Münze zu verwandeln. Die protestantische Religion steht für diese Rettung der Utopie vor ihrer Verwirklichung. Nur so gewinnen wir ihre inspirierende Kraft wieder.

\section{Die Gottesgeschichte und die Geschichten der Menschen}

Wir stehen gegenwärtig in einer heftigen Kontroverse um die Fragen einer angemessenen Bioethik. Postmodernistische Ethiker reden in diesem Zusammenhang einer Pluralisierung der Ethik das Wort. Im Grunde sollten wir - so wird uns geraten - bereits den Singular Ethik vergessen und nur noch von Ethiken sprechen. Den in Fragen der Bioethik eher Zögerlichen und Skeptischen wird vorgehalten, an einem überholten, christlich-kantianischen' Menschenbild fest zu halten, das sich durch die Auswüchse der Moderne desavouiert habe. Dem wird dann gerne - so etwa durch Peter Sloterdijk - eine von Nietzsches Lebensphilosophie inspiriertes Ethiken-Ensemble entgegengehalten.

Ich halte diese gegenwärtige Kontroverse um die Bioethik für zeitdiagnostisch höchst aufschlussreich. Eine Pluralisierung der Ethik wird gegen bestimmte Formen traditioneller Ethik eingeklagt. In gewisser Hinsicht stimme ich mit diesem Postulat überein. Wir werden auch in Fragen der Ethik nicht umhin kommen, festzustellen, dass wir es heute mit einem Markt höchst verschiedener, heterogener Ethiken zu tun haben. Dahinter können und sollten wir auch nicht zurückgehen. Was ich allerdings für widersprüchlich halte, ist der Umstand, dass nun doch gerade die Vertreter postmoderner Ethik-Konzeptionen sagen, eine bestimmte Form - nämlich die nicht-plurale Form - der Ethik ist überholt. Im Grunde sind diese Vertreter immer noch der klassischen Moderne und ihrem Fortschrittsdenken verpflichtet. Pluralität ist in dieser Perspektive ,moderner' als Singularität. Im Grunde zeigt sich aber sehr schnell, dass nicht singulare und plurale Ethiken gegenwärtig miteinander konkurrieren, sondern verschiedene Ethiken, die Bezug nehmen auf bestimmte Menschenbilder und auf die diese Menschenbilder basal begleitenden $\mathrm{Ge}$ schichten. Gerade wer sich auf Nietzsche beruft, sollte diesen Sachverhalt im Blick haben. Es sind die basalen Geschichten von Menschen, die heute miteinander in Konflikt geraten, und weniger die daraus abgeleitetet Ethiken.

Protestantische Religion bringt nun in das gegenwärtige Konzert der basalen Geschichten vom Menschen ihre bestimmte Geschichte ein, nämlich die Gottesgeschichte mit Israel und in Jesus Christus mit der ganzen Menschheit. Mit dieser Gottesgeschichte und den damit verbundenen Menschengeschichten werden Theologie und Kirche in den Kontroversen unserer kulturellen Ge- 


\section{Thema: Säkularisierung}

genwart kenntlich. Dabei können diese Geschichten gerade für die postmodernen Menschen ihre besondere Attraktivität gewinnen. Denn diese Geschichten erzählen vom schwachen Subjekt, von der menschlichen Existenz als Fragment, von unserer Verschlungenheit und Verstricktheit in ambivalente Verhältnisse. Alles Dinge, die den postmodernen Menschen alltäglich vertraut sind. Und so ist es alles andere als ein Zufall, dass jüngst der graubündische Schriftsteller Iso Camartin ein Loblied auf diesen besonderen Bestand an menschheitlichen Geschichten angestimmt hat: "So ist die Bibel für mich das große Wunderbuch der schönen und fesselnden Ungereimtheiten geworden ... [Ich sehe] die Bibel als einen riesigen Wörterbaum ..., unter den man sich legt, um tausend Zweige und Triebe in ihrem Wachstum und ihrem Wildwuchs zu verfolgen. Was für überraschende Dinge man da entdeckt! Auf jedem Ast, in jedem Zweig schimmert etwas im Licht der eigenen Neugierde ... [Für mich] ist die Bibel nicht, gebietende Lehre' und doch Quelle großer Offenbarungen. Sie ist Geschichtsbuch und Poesiebuch, reiche Sammlung alter Erzählungen und unerschöpfliches Reservoir der schönsten Bilder, zu denen Sprache überhaupt fähig ist ... Da staunt der Leser über den Reichtum an Beobachtung, Lebenserfahrung, ja Klugheit, der ihm aus der Bibel entgegenkommt ... Solche Geschichten haben kein Ende. Man muss immer wieder zu ihnen zurück ... Das sind nicht irgendwelche exotischen Ereignisse und Örtlichkeiten in einem früheren Mesopotamien. Es sind Schlüsselerlebnisse im Leben eines jeden Lesers, der die Welt verstehen will."

\section{Frei-Raum des Individuums}

Ich komme zum letzten Punkt meines Profils protestantischer Religion für die Postmoderne angesichts der Rückkehr des Heiligen in die Stadt ohne Gott. Die Menschen der Postmoderne müssen heute ihre Lebensentwürfe selbst wählen und konstruieren. Nur eines können sie nicht mehr wählen, nämlich dass sie wählen müssen. Deshalb ist diese Wahl nicht nur Freiheit, sondern stets auch Zwang. Es ist eine Freiheit, die nicht gewählt werden kann, eine Freiheit folglich mit Zwangscharakter. Dieser Zwang überfordert Menschen nicht selten. Inmitten dieser Konstellation erzählt protestantische Religion eine merkwürdig fremde Geschichte. Sie erzählt davon, dass wir Menschen vor all unserer Wahl bereits von Gott erwählte Menschen sind. Dass wir uns nicht durch unsere Wahl konstituieren, sondern dass wir vor allem Wählen bereits schon konstituiert sind. Eine merkwürdige Geschichte für die Ohren postmoderner Menschen. Eine Geschichte, die nicht einfach in der vorfindlichen Lebenserfahrung zu entdecken ist, sondern die vielleicht erst, nachdem sie uns als fremde Geschichte erzählt wurde, auch von uns in unserem Leben als wahre Geschichte entdeckt werden kann. Mit dieser fremden Geschichte schafft protestantische Religion einen spezifischen religiösen begründeten Frei-Raum. Dieser Frei-Raum wird nur erfahrbar im Lichte dieser erzählten

5 Iso Camartin: Die Bibliothek von Pila, Frankfurt 1997, $27 \mathrm{f}$. 
fremden Geschichte. Gehört wird diese fremde Geschichte am besten dort, wo wir sie als eine Geschichte erzählen, die dem einzelnen, unverwechselbaren, individuellen Menschen zugute kommt. Die protestantische Leidenschaft für den Einzelnen, für das Individuum, die dem Protestantismus im Laufe seiner Geschichte nicht selten zum Vorwurf gemacht wurde, diese Leidenschaft für den und die Einzelne kann dem Protestantismus in unserer kulturellen Gegenwart nur zugute kommen. Protestantische Religion steht für die Wahrung eines religiösen Frei-Raums, der alle Ansprüche einer kritischen Überprüfung unterzieht. Nicht ein Theologe, sondern der Religionssoziologe Hans-Georg Soeffner hat diese Herausforderung für die protestantische Religion auf den Begriff gebracht. Deshalb möchte ich ihn zum Abschluss zitieren: Die christliche Botschaft kann "weder eine Verdoppelung politischer Rede im kirchlichen Raum noch die Ausbildung von Sprachspielen für geistliche Reservate oder esoterische Cliquen meinen. Auch und gerade da, wo Kirche Minderheiteninteressen und unterdrückte Meinungen vertritt, legitimiert sie sich nicht durch ein pragmatisches Partialinteresse. Ihre universalistische Legitimation gewinnt sie eben dadurch, dass sie die kleinste gesellschaftliche Minderheit, jedes einzelne Individuum und dessen Ängste und Hoffnungen, gegen normative Ansprüche, gleich welcher Gesellschaften, Verbände oder Gruppen verteidigt. Insofern konfrontiert sie die Haltung ihres Religionsstifters mit dem Gruppen- oder Kollektivinteresse, mit Karl Barth gesprochen: Das Offenbarungswort mit dem ,Zeit-Wort'." ${ }^{17}$

Hans-Georg Soeffner: Was kann die moderne Gesellschaft von der Predigt erwarten?, in: Dokumentation des Societas Homiletica Congress II, Berlin 1995, 7-17, zit. 17.

\section{Fremde Heimat Religion}

Konturen kirchlichen Lebens in 0stdeutschland ${ }^{1}$

Jan Hermelink

\section{Fremd im eigenen Land: Zu den (kirchlichen) Verhältnissen in Ostdeutschland}

Die folgende Skizze muss sich von vornherein auf einige Bedenken einstellen. Abgesehen von dem Generalverdacht, eine westdeutsche Perspektive könne hier ohnehin nur verzerren, lässt sich gegen den Versuch, die gegenwärtige kirchliche Situation im Gebiet der ehemaligen DDR darzustellen, zunächst die große Vielfalt der Verhältnisse ins Feld führen: Nord und Süd, lutherische und

\footnotetext{
Bearbeitung eines Vortrages über die "Kirchliche Situation in Ostdeutschland“ gehalten am i . Juni 2001 im Evangelischen Stift Tübingen.
} 